\title{
Title: $\quad$ The Truth about Trauma Readmissions
}

Authors: $\quad$ Olubode A. Olufajo, MD, MPH ${ }^{\mathrm{a}, \mathrm{b}}$ - oao777@mail.harvard.edu

Zara R. Cooper, MD, MSc ${ }^{\text {a, b }}$ - zcooper@partners.org

Brian K. Yorkgitis, DO ${ }^{\text {a }}$ - byorkgitis@ partners.org

Peter A. Najjar, MD ${ }^{\mathrm{b}}$ - pnajjar@ partners.org

David Metcalfe, LLB, MBChB ${ }^{\mathrm{b}}$ - d.metcalfe@ doctors.org.uk

Joaquim M. Havens, MD ${ }^{\mathrm{a}, \mathrm{b}}$ - jhavens@ partners.org

Reza Askari, MD ${ }^{\text {a, b }}$ - raskari@ partners.org

Gabriel A. Brat, MD, MSc ${ }^{\mathrm{a}}$ - gbrat@ partners.org

Adil H. Haider, MD, MPH ${ }^{\text {a }, ~ b ~}$ - ahhaider@partners.org

Ali Salim, MD ${ }^{\text {a, b }}$ - asalim1@ partners.org

a. Division of Trauma, Burn and Surgical Critical Care

Department of Surgery

Brigham and Women's Hospital

75 Francis Street

Boston, MA 02115, USA

b. Center for Surgery and Public Health

Brigham and Women's Hospital

Harvard Medical School and Harvard T.H. Chan School of Public Health 1620 Tremont Street

Boston, MA 02121, USA

Corresponding Author:

Olubode A. Olufajo, MD, MPH

Division of Trauma, Burn and Surgical Critical Care

Department of Surgery

Brigham and Women's Hospital

75 Francis Street

Boston, MA 02115, USA

Tel: (617) 525-7300, Fax: (617) 566-9549

Email: oao777@mail.harvard.edu 


\section{INTRODUCTION}

Traumatic injuries exert an economic burden of more than $\$ 406$ billion annually in the United States.(1) Up to 2.3 million hospital admissions are primarily due to trauma,(2) which is higher than admissions due to stroke and cancer combined. $(3,4)$ The increased efficiency of trauma systems and the advancement of medical technology have led to consistent decreases in mortality in these patients over the last few years and up to $6 \%$ decrease in mortality was seen between 2002 and 2010.(5) With improved survival of trauma patients has come the attendant focus on reducing morbidity in these patients. This has led to increasing interest in various metrics to assess the quality of care patients receive other than hospital mortality.(6-8) These metrics include complication rates, length of hospital stay, and hospital readmission.

Hospital readmission has been commonly used as a quality measure by hospitals and government agencies over the past decade. The popularity of this measure increased with the Hospital Readmission Reduction Program (HRRP) introduced by the Affordable Care Act of 2010.(9) This program penalized hospitals with excess readmissions for certain conditions by reducing their Medicare reimbursements, with an aim to encourage improvements. The obvious financial implications of this triggered the creation of numerous strategies to reduce avoidable readmissions to individual hospitals. An example of this is the recommendation based on costeffectiveness analysis that more surgical patients should be treated on out-patient bases instead of being readmitted.(10) Although the HRRP is restricted to few non-surgical conditions, there is a possibility that it will involve surgical conditions in the near future.(11) This highlights the need for pro-active measures to reduce readmissions in trauma patients.

Despite the fact that some efforts are being made to reduce readmissions among trauma patients, there is little that is known about the actual readmission rates in these patients. Most of the 
estimates commonly referenced are among patients with specific injuries or age ranges and cannot be applied to the general trauma population. $(12,13)$ In addition, most studies assess readmissions to their own institutions alone or to a small network of hospitals.(14-16) Because patients may be readmitted at other hospitals, these studies do not accurately assess readmissions.(17) Furthermore, in order to identify areas to target interventions and to stratify patients based on their risk of readmission, it is important to assess the patient factors that are associated with readmission among trauma patients. Some studies have highlighted these factors among other patient populations.(18) However, information on US trauma patients is lacking. Therefore, to accurately determine the readmission rates and characteristics of readmission among a representative sample of trauma patients, we examined hospital discharge records from a statewide database.

\section{METHODS}

\section{Selection of Study Population}

We examined hospital discharge records from 2007 - 2011 in the California State Inpatient Database (SID), Healthcare Cost and Utilization Project (HCUP), Agency for Healthcare Research and Quality. This database contains hospital records from up 98\% of the hospitals in the state.(19) It also contains unique patient identifiers which are independent of the hospitals that patients are admitted enabling patient records to be linked over time.

We identified patients of age $18-64$ years that had the International Classification of Disease- $9^{\text {th }}$ revision (ICD-9) diagnosis codes corresponding to traumatic injuries. ICD-9 diagnosis codes 800 - 904, $910-929$, and 950 - 959 were used to define the study cohort. Patients that died during 
admission, those that were transferred to other hospitals, those with isolated hip fractures, and those that were admitted or discharged in the last month of available data were not included in the final cohort.

\section{$\underline{\text { Patient Characteristics }}$}

We included data on patients' age (18-44, $\geq 45)$, sex, and race (White, Black, Hispanic, Asian/Pacific Islander, Native American and Others). Insurance type was classified as public, private, self-pay, and others. “Others” included Worker's Compensation, County Indigent Programs, Other Government aids and Other Indigent funds.(20)

The Charlson Co-morbidity Index, which is a weighted score ranging from 0 to 37 with a higher score indicating greater co-morbidity,(21) was used to assess the degree of co-morbidity of the patients. We calculated this using the CHARLSON command in Stata.(22) Charlson scores were classified as $<2$ and $\geq 2$.

$\underline{\text { Injury and Admission Characteristics }}$

Injury Severity Scores (ISS) derived from ICD-9 diagnosis codes were calculated for the patients. The method of estimation has been previously described and validated.(23, 24) We used the ICDPIC program in Stata to calculate the scores.(25) Scores were classified as $<9,9-15,16$ 24 , and $\geq 25$. Using the Abbreviated Injury Scale for each body region, which is an anatomicalbased injury severity measure,(26) we determined the region of maximum injury severity. We classified regions as Head/Neck/Face, Thorax, Abdomen/pelvic organs, Extremities/Limb girdles, and External. 
Diagnoses made during admission that were not present at the time of admission were classified as complications during admission. The length of hospital stay was categorized as 0-3, 4-7, and 77. Patients' discharge dispositions were categorized as Home, Home Health Care, Skilled Nursing Facility (SNF)/Intermediate Care Facility (ICF)/Other Facilities, and Against Medical Advice (AMA).

$\underline{\text { Readmission Rates and Readmission Characteristics }}$

The 30-day readmission rate was defined as the proportion of patients that had unplanned readmissions within 30 days after discharge among the patients that were discharged. The reasons for readmission were determined by the primary admitting ICD-9 diagnosis codes. These reasons were classified using categories previously described.(27) We included two other categories in order to capture more diagnoses; musculoskeletal conditions and psychiatric conditions.

Among the patients that were readmitted, we determined those that returned to the same hospitals and those that went to other hospitals. Then, we evaluated some common measures of patient outcomes during their readmission such as in-hospital mortality, complication rate and length of stay. We also determined the proportion of patients that were readmitted within 30 days of discharge from their first readmission.

\section{$\underline{\text { Statistical Analysis }}$}

The proportions of patients in the different patient groups were determined. Patient, injury and admission characteristics were compared among different groups using Chi-square tests for categorical variables and Wilcoxon rank-sum tests for continuous variables. The risk factors for readmission were evaluated by univariate logistic regression models. Factors that met the $P<0.1$ 
level of significance on univariate analyses were used in building a multivariate logistic regression model to determine independent risk factors for 30-day readmission. All analyses were done using Stata Statistical Software: Release 13 (College Station, TX: StataCorp LP) and the significance level was set as $P<0.05$.

\section{RESULTS}

\section{$\underline{\text { Patient, Injury and Admission Characteristics }}$}

Our final cohort consisted of 252,752 patients admitted with diagnoses of traumatic injuries. They were mostly male $(66.79 \%)$, white $(58.12 \%)$, and less than 45 years old $(53.15 \%)$ (Table 1). The predominant insurance type was private insurance (40.77\%) and only $6.36 \%$ had Charlson Scores $\geq 2$. The injury pattern was mostly mild with $11.76 \%$ of patients having ISS $>15$. The regions of maximum severity were more frequently the extremities/limb girdles (44.87\%) and the head/neck/face $(28.92 \%)$.

Majority of the patients had hospital lengths of stay less than 4 days (86.10\%) and complications during admission were seen in $14.47 \%$ of patients. Patients were discharged home in most cases (80.87\%) and $1.78 \%$ left AMA.

\section{$\underline{\text { Readmission Rates and Risk of Readmission }}$}

The overall 30-day readmission rate was $7.56 \%$. There were wide variations seen in readmission rates among the different patient groups (Table 1). Patients with Charlson Scores $\geq 2(17.75 \%)$, those discharged AMA (17.12\%), and those on public insurance (11.74\%) had significantly higher readmission rates than other patients. Other groups with high readmission rates were those 
with hospital lengths of stay greater than 3 days, those discharged to SNF, ICF or Home Health Care, and those of Native American descent.

On multivariate analyses, the strongest independent predictors of 30-day readmission were being discharged AMA [adjusted Odds Ratio (aOR): 2.56 (95\% Confidence Interval, 2.35 - 2.78)] and Charlson Scores $\geq 2$ [aOR: $2.05(1.96-2.16)$ ] (Table 2). Male patients [aOR: 1.14 (1.10 $1.18)$ ], patients $\geq 45$ years old [aOR: $1.32(1.27-1.36)$ ], and those that were not on private insurance had higher risks of readmission. Compared to patients with head/neck/face injuries, patients with maximum injury severity in the abdomino-pelvic region [aOR: $1.28(1.21-1.36)$ ] and extremities/limb girdles [aOR: $1.22(1.17-1.27)]$ were more likely to be readmitted within 30 days.

\section{$\underline{\text { Reasons for Readmission and Readmission Characteristics }}$}

Most patients that were readmitted had primary readmission diagnoses of musculoskeletal complaints as defined by ICD-9 diagnosis codes (22.29\%). Significant proportions were readmitted for psychiatric conditions (9.40\%) and surgical infections (6.69\%). Other major reasons for readmission were pulmonary, gastrointestinal and genitourinary conditions (Figure 1).

Among patients that were readmitted, $34.5 \%$ were readmitted to hospitals different from the hospitals they received their initial care. There were significant differences in the outcomes among patients that were readmitted to the same hospital and those that were readmitted to different hospitals (Table 3 ). The in-hospital mortality ( $0.78 \%$ vs. $1.21 \%, P=0.003)$, mean lengths of stay (5.38 days vs. 5.99 days, $P<0.005)$ and probability of second readmissions $(16.27 \%$ vs. $20.74 \%, P<0.005)$ were better in those that were readmitted to the same hospital 
compared to those that were readmitted to different hospitals. There were no significant differences in the complication rates based on where patients were readmitted $(P=0.875)$.

\section{DISCUSSION}

Benchmarking of trauma outcomes including readmissions is essential for comparing hospitals based on their measured performance.(6) In an era where public reporting of outcomes is fast becoming the standard practice, it is important to accurately estimate the expected outcomes for the care of unique patient groups.(28) Our study showed that $7.56 \%$ of trauma patients had unplanned readmissions within 30 days of discharge. Given the large amount of resources that go into every hospitalization and the higher morbidity associated with readmissions, $(29,30)$ this proportion of patients represents a potential area for improvement in the care of trauma patients. Identification of factors that lead to these readmissions is a key to formulating strategies to address them.

Prior studies in subsets of trauma patients found risk factors for readmission similar to those seen in our study.(13, 14, 16, 31, 32) Significant risk factors for readmission including male sex, older age, being on public insurance, having higher injury scores, longer hospital stays, higher comorbidity scores, and being discharged AMA have also been shown in other studies. These factors indicate that apart from the care patients receive, specific patient characteristics may be involved in their readmissions. This is particularly noteworthy in the light of hospital comparisons based on patient outcomes. The Center for Medicare and Medicaid Services only adjusts for limited patient characteristics when making comparisons of hospital performance.(9) If these sorts of comparisons become the standards for examining trauma readmission, it will be 
important to consider the patient mix in order not to unfairly blacklist hospitals that care for disadvantaged patients. $(11,33)$

Although one-fifth of the patients were readmitted for musculoskeletal reasons likely directly related to their initial injury, a large proportion of patients had diagnoses that may represent some underlying vulnerabilities. Psychiatric conditions are diagnoses that have been cited as major reasons for readmission in trauma patients.(18) The fact that these conditions can often be assessed before discharge and that they may have been associated with the initial injury highlights the need for increased surveillance for these conditions among trauma patients prior to discharge. Early interventions including multidisciplinary approaches may help to reduce these preventable readmissions and improve patients' wellbeing. Like other studies, $(15,31)$ surgical infections formed a significant proportion of readmissions in our cohort. A study of readmissions due to surgical site infections (SSIs) showed that more than half of patients diagnosed with SSIs after discharge were readmitted and that the readmission rates in patients diagnosed after discharge was up to 7 times higher than the rates in patients diagnosed before discharge.(34) The use of checklists prior to discharge, early follow up calls by dedicated health workers, and scheduling of early clinic visits are ways that have been suggested to reduce readmissions due to surgical infections.(34)

One of the advantages of our study is that we included hospital readmission to hospitals other than where index hospitalizations occurred. The finding that $34.5 \%$ of readmitted patients were readmitted to different hospitals brings to fore the inaccuracies in same-hospital readmission rates. This may explain why the readmission rates we found were slightly higher than that seen in other studies. $(15,31)$ It also highlights the level of fragmentation of patient care seen in trauma surgery. Fragmentation of care has been previously associated with poorer patient 
outcomes.(35) Our study showed that patients that were readmitted to different hospitals had higher in-hospital mortality rates, longer lengths of stay, and higher rates of subsequent readmissions. This is indicative of the need for efforts directed towards continuity and integration of patient care including increased communication between care providers.

Our study has some limitations. First, we used an administrative database and had only limited details of patients' clinical characteristics. Because some of these characteristics may be related to hospital readmission, we may have not have fully captured the risk factors for hospital readmission. However, it is unlikely that these characteristics will be relevant for risk-adjustment in programs that compare hospitals based on their readmission rates. In addition, we did not include patients older than 64 years in our analyses meaning the results of our study are not generalizable to older patients. Because the physiology and mechanisms of injury differ significantly in older patients compared to younger patients,(36-38) we decided it will be more appropriate to analyze this group of patients separately.

\section{$\underline{\text { Conclusion }}$}

Hospital readmission among trauma patients has immense clinical and cost implications. The efforts currently being made to reduce readmissions will be more successful with an in-depth understanding of the factors that surround readmissions in this group of patients. Providing assistance to socially vulnerable patients, early identification of potential complications that could lead to readmission and increasing integration of patient care are areas that could be focused on to reduce readmissions among trauma patients. 


\section{$\underline{\text { References }}$}

1. Finkelstein EA, Corso PS, Miller TR. The incidence and economic burden of injuries in the United States: Oxford University Press; 2006.

2. National Trauma Institute. Trauma Statistics. National Trauma Institute; 2014 [updated February 2014; cited 2015 April 7]; Available from: http://www.nationaltraumainstitute.org/home/trauma_statistics.html.

3. Centers for Disease Prevention and Control. Cerebrovascular Disease or Stroke. Atlanta, GA: CDC/National Center for Health Statistics; 2013 [updated February 06, 2015; cited 2015 April 07]; Available from: http://www.cdc.gov/nchs/fastats/stroke.htm.

4. Hall MJ, DeFrances CJ, Williams SN, et al. National hospital discharge survey: 2007 summary. Natl Health Stat Report. 2010;29(29):1-20.

5. Sise RG, Calvo RY, Spain DA, et al. The epidemiology of trauma-related mortality in the United States from 2002 to 2010. Journal of trauma and acute care surgery. 2014;76(4):913-20. 6. Hashmi ZG, Schneider EB, Castillo R, et al. Benchmarking trauma centers on mortality alone does not reflect quality of care: implications for pay-for-performance. Journal of Trauma and Acute Care Surgery. 2014;76(5):1184-91.

7. Moore L, Stelfox HT, Boutin A, Turgeon AF. Trauma center performance indicators for nonfatal outcomes: A scoping review of the literature. Journal of Trauma and Acute Care Surgery. 2013;74(5):1331-43.

8. Moore L, Stelfox HT, Turgeon AF, et al. Derivation and Validation of a Quality Indicator of Acute Care Length of Stay to Evaluate Trauma Care. Annals of surgery. 2014;260(6):1121-7. 9. Centers for Medicare and Medicaid Services. Readmissions Reduction Program Baltimore, MD: Centers for Medicare \& Medicaid Services; 2014 [updated August 4, 2014; cited 
2015 February 15]; Available from: http://www.cms.gov/Medicare/Medicare-Fee-for-ServicePayment/AcuteInpatientPPS/Readmissions-Reduction-Program.html.

10. Postel M, Frank PN, Barry T, et al. The Cost of Preventing Readmissions: Why Surgeons Should Lead the Effort. The American Surgeon. 2014;80(10):1003-6.

11. Shih T, Ryan AM, Gonzalez AA, Dimick JB. Medicare's Hospital Readmission Reduction Program in Surgery May Disproportionately Affect Minority-Serving Hospitals. Annals of surgery. 2014.

12. Spector WD, Mutter R, Owens P, Limcangco R. Thirty-day, all-cause readmissions for elderly patients who have an injury-related inpatient stay. Medical care. 2012;50(10):863-9. 13. Fawcett VJ, Flynn-O'Brien KT, Shorter Z, et al. Risk factors for unplanned readmissions in older adult trauma patients in Washington state: a competing risk analysis. Journal of the American College of Surgeons. 2014.

14. Marcin JP, Romano PS. Impact of between-hospital volume and within-hospital volume on mortality and readmission rates for trauma patients in California*. Critical care medicine. 2004;32(7):1477-83.

15. Morris DS, Rohrbach J, Sundaram LMT, et al. Early hospital readmission in the trauma population: Are the risk factors different? Injury. 2014;45(1):56-60.

16. Vachon CM, Aaland M, Zhu TH. Readmission of trauma patients in a nonacademic Level II trauma center. Journal of Trauma and Acute Care Surgery. 2012;72(2):531-6.

17. Gonzalez AA, Shih T, Dimick JB, Ghaferi AA. Using Same-Hospital Readmission Rates to Estimate All-Hospital Readmission Rates. Journal of the American College of Surgeons. 2014;219(4):656-63. 
18. Moore L, Stelfox HT, Turgeon AF, et al. Rates, patterns, and determinants of unplanned readmission after traumatic injury: a multicenter cohort study. Annals of surgery. 2014;259(2):374-80.

19. Healthcare Cost and Utilization Project. Overview. Rockville, MD: Healthcare Cost and Utilization Project; 2015 [updated November 2014; cited 2015 Mar 20]; Available from: http://www.hcup-us.ahrq.gov/sidoverview.jsp.

20. Healthcare Cost and Utilization Project. SID Documentation. Rockville, MD: Healthcare Cost and Utilization Project; 2014 [updated August 2008; cited 2015 Mar 20]; Available from: http://www.hcup-us.ahrq.gov/db/vars/siddistnote.jsp?var=pay1.

21. Charlson ME, Pompei P, Ales KL, MacKenzie CR. A new method of classifying prognostic comorbidity in longitudinal studies: development and validation. Journal of chronic diseases. 1987;40(5):373-83.

22. Stagg V. CHARLSON: Stata module to calculate Charlson index of comorbidity. Statistical Software Components. 2006.

23. Osler T, Rutledge R, Deis J, Bedrick E. ICISS: an international classification of disease-9 based injury severity score. Journal of Trauma and Acute Care Surgery. 1996;41(3):380-8.

24. Rutledge R, Osler T, Emery S, Kromhout-Schiro S. The end of the Injury Severity Score (ISS) and the Trauma and Injury Severity Score (TRISS): ICISS, an International Classification of Diseases, ninth revision-based prediction tool, outperforms both ISS and TRISS as predictors of trauma patient survival, hospital charges, and hospital length of stay. Journal of Trauma and Acute Care Surgery. 1998;44(1):41-9. 
25. Clark DE, Osler TM, Hahn DR. ICDPIC: Stata module to provide methods for translating International Classification of Diseases (Ninth Revision) diagnosis codes into standard injury categories and/or scores. Statistical Software Components. 2010.

26. Palmer CS, Lang J, Russell G, et al. Mapping abbreviated injury scale data from 1990 to 1998 versions: a stepping-stone in the contemporary evaluation of trauma. Injury. 2013;44(11):1437-42.

27. Kassin MT, Owen RM, Perez SD, et al. Risk factors for 30-day hospital readmission among general surgery patients. Journal of the American College of Surgeons. 2012;215(3):32230.

28. Minami CA, Dahlke A, Bilimoria KY. Public Reporting in Surgery: An Emerging Opportunity to Improve Care and Inform Patients. Annals of surgery. 2015;261(2):241-2. 29. Tsai TC, Joynt KE, Orav EJ, et al. Variation in surgical-readmission rates and quality of hospital care. New England Journal of Medicine. 2013;369(12):1134-42.

30. Jencks SF, Williams MV, Coleman EA. Rehospitalizations among patients in the Medicare fee-for-service program. New England Journal of Medicine. 2009;360(14):1418-28.

31. Battistella FD, Torabian SZ, Siadatan KM. Hospital readmission after trauma: an analysis of outpatient complications. Journal of Trauma and Acute Care Surgery. 1997;42(6):1012-7. 32. Laupland KB, Svenson LW, Grant V, et al. Long-term mortality outcome of victims of major trauma. Injury. 2010;41(1):69-72.

33. Haider AH, Weygandt PL, Bentley JM, et al. Disparities in trauma care and outcomes in the United States: a systematic review and meta-analysis. The journal of trauma and acute care surgery. 2013;74(5):1195. 
34. Gibson A, Tevis S, Kennedy G. Readmission after delayed diagnosis of surgical site infection: a focus on prevention using the American College of Surgeons National Surgical Quality Improvement Program. The American Journal of Surgery. 2014;207(6):832-9.

35. Tsai TC, Orav EJ, Jha AK. Care Fragmentation in the Postdischarge Period: Surgical Readmissions, Distance of Travel, and Postoperative Mortality. JAMA surgery. 2014.

36. Keller JM, Sciadini MF, Sinclair E, O'Toole RV. Geriatric trauma: demographics, injuries, and mortality. Journal of orthopaedic trauma. 2012;26(9):e161-e5.

37. Bennett KM, Scarborough JE, Vaslef S. Outcomes and health care resource utilization in super-elderly trauma patients. Journal of Surgical Research. 2010;163(1):127-31.

38. Kuhne CA, Ruchholtz S, Kaiser GM, Nast-Kolb D. Mortality in severely injured elderly trauma patients—-when does age become a risk factor? World journal of surgery. 2005;29(11):1476-82. 